\title{
Protegiendo los derechos de propiedad intelectual y el conocimiento ecológico tradicional: una mirada crítica a la Ley 27811 del Perú*
}

\section{Michelle L. Hak Hepburn}

iD https://orcid.org/0000-0001-9113-7309

Universidad de Columbia Británica

mhakhep@student.ubc.ca

\section{PRESENTACIÓN}

El siguiente artículo, originalmente escrito y publicado en inglés, analiza la implementación comunitaria de una ley peruana, la Ley 27811: Régimen de Protección de los Conocimientos Colectivos de los Pueblos Indígenas vinculados a los Recursos Biológicos. Se pretende analizarlo bajo un marco decolonial; sin embargo, el hecho de solo aparecer en inglés cuando se trata de trabajos realizados en el Perú reproduce la colonialidad y jerarquía de conocimientos académicos. Es importante que un análisis de la implementación de una ley peruana que busca proteger a los conocimientos de los pueblos indígenas esté disponible en castellano, para informar acerca de los trabajos futuros relacionados con la Ley 27811, entre otras intervenciones, para apoyar a los pueblos originarios en Perú y en América Latina. Adicionalmente, el artículo contribuye a las conversaciones académicas de las relaciones entre el Estado y los indígenas y de las concurrencias y disyunciones de sus miradas al territorio y los «recursos» biológicos.

* Versión original en inglés, reproducida con permiso de la Sociedad de Antropología Aplicada: Hak Hepburn, M (2020). Protecting Intellectual Property Rights and Traditional Ecological Knowledge: A Critical Look at Peru's Law 27811. Human Organization 79(1), 69-79. Traducción de Daniela Velasco, Universidad de Columbia Británica. https://orcid.org/00000002-5425-9240 
RESUMEN

La Ley 27811 del gobierno peruano es una ley sobre propiedad intelectual aprobada en 2002 y diseñada para registrar y proteger la sabiduría tradicional. Brinda oportunidades productivas para elaborar un análisis crítico y está formulada dentro de la trayectoria de los derechos de propiedad intelectual internacionales y discusiones que dificultan la integración de los conocimientos ecológicos tradicionales (CET) a los marcos de referencia científicos cartesianos. Este documento examina de manera crítica cómo ha sido implementada esta ley peruana y los impactos que tiene en las comunidades indígenas, particularmente en la alta Amazonía. El análisis está basado en el trabajo de la autora al asistir a las comunidades indigenas en San Martín a registrar sus conocimientos por medio de esta ley. Mientras que la ley representa un acercamiento legal avanzado para abordar las desigualdades del poder, sigue siendo problemática: no aborda el empobrecimiento de la población indigena y continúa subordinando los CET indigenas a la ciencia cartesiana. Aunque sí representa un reconocimiento simbólico del valor de los pueblos indigenas peruanos, aún son necesarios otros mecanismos para poder compensar la larga historia de colonización y racismo.

Palabras clave: propiedad intelectual, conocimiento ecológico tradicional, Perú, pueblos indígenas, bioprospección.

\section{Protecting Intellectual Property Rights and Traditional Ecological Knowledge: A Critical Look at Peru's Law 27811}

ABSTRACT

The Peruvian government's Law N. 27811, an intellectual property law passed in 2002 and designed to register and protect traditional knowledge, provides productive opportunities for critical analysis. Framed within the trajectory of international intellectual property rights and discussions that complicate the integration of Traditional Ecological Knowledge (TEK) into Cartesian scientific frameworks, this paper critically examines how the Peruvian law 
has been implemented and its impacts in Indigenous communities, particularly in the Andean Amazon region. The analysis is based on the author's work assisting Indigenous communities in San Martin register their knowledge through this law. While the law represents an advanced legal attempt to address power inequalities, it remains problematic. It does not address the impoverishment of Indigenous Peoples and continues to subordinate Indigenous TEK to Cartesian science. Although it is a symbolic recognition of the value of Peruvian Indigenous Peoples, other mechanisms are still required to redress the long history of colonization and racism.

Keywords: intellectual property, traditional ecological knowledge, Peru, Indigenous Peoples, bioprospecting. 


\section{INTRODUCCIÓN}

En un esfuerzo por proteger los derechos de los indígenas y el patrimonio nacional, en 2002 se aprobó en Perú la Ley 27811, Régimen de Protección de los Conocimientos Colectivos de los Pueblos Indígenas vinculados a los Recursos Biológicos. Los derechos de propiedad intelectual (DPI) restringen el uso de sabidurías y productos creados por otros.

Los investigadores y las organizaciones transnacionales utilizan los sistemas de DPI convencionales - incluyendo las patentes y los derechos de autor-para asegurar ganancias, aunque a veces las patentes estén basadas en conocimientos obtenidos de los pueblos indígenas. Alrededor del mundo, primero los colonizadores se apoderaron la tierra, y los investigadores continúan apoderándose del conocimiento de los pueblos originarios (LaDuke, 2005; Shiva, 2001; Tuhiwai Smith, 2012). A pesar de que los DPI fueron «construidos juntos con los derechos generales de la soberanía territorial y de recursos», en la década de 1990 (Varese, 1996, p.136), los pueblos indígenas comenzaron a separar los DPI de los derechos sobre la tierra para obtener mayor impulso internacional (Muehlebach, 2011). Para defenderse de las apropiaciones culturales, a menudo la cultura es tratada como propiedad, lo que impone limitaciones sobre quienes buscan proteger sus derechos (Coombe, 1998; Greene, 2004).

Los objetivos de la Ley 27811 se describen en el artículo 5:

1) Promover el respeto, la protección, la preservación, la aplicación más amplia y el desarrollo de los conocimientos colectivos de los pueblos indígenas;

2) Promover la distribución justa y equitativa de los beneficios derivados de la utilización de estos conocimientos colectivos;

3) Promover el uso de estos conocimientos en beneficio de los pueblos indígenas y de la humanidad;

4) Garantizar que el uso de los conocimientos colectivos se realice con el consentimiento informado previo de los pueblos indígenas; 
5) Promover el fortalecimiento y el desarrollo de las capacidades de los pueblos indígenas y de los mecanismos tradicionalmente empleados por ellos para compartir y distribuir beneficios generados colectivamente, en el marco del presente régimen;

6) Evitar que se concedan patentes a invenciones obtenidas o desarrolladas a partir de conocimientos colectivos de los pueblos indígenas del Perú, sin que se tomen en cuenta estos conocimientos como antecedentes en el examen de novedad y nivel inventivo de dichas invenciones.

Los expertos en el ámbito jurídico consideran a la Ley 27811 como un instrumento bien desarrollado para proteger los DPI de los pueblos indígenas peruanos (Clark, Lapeña y Ruíz, 2004; Taubman y Leistner, 2008), pero sus impactos y sus procedimientos de implementación basados en la comunidad aún no se han examinado. Las protecciones legales pueden ser un reconocimiento simbólico importante para los pueblos indígenas. Sin embargo, las leyes simbólicas no cambian las relaciones coloniales entre el Estado y los pueblos originarios y pueden reforzar categorías de reconocimiento impuestas por el Estado (Coulthard, 2014; Drahos, 2014). El análisis de la implementación y los impactos de dicha ley es crucial para evaluar si este tipo de protección es puramente simbólica. Por consiguiente, presento un análisis crítico y teórico de la Ley 27811 basado en mi experiencia de su implementación y evaluándolo a través de un marco de referencia decolonial.

Los eruditos en el ámbito decolonial ubican el surgimiento de la modernidad al inicio de la colonización de las Américas (Asher, 2013; Mignolo, 2011; Quijano, 2000). Las estructuras de los poderes nacionales en las Américas fueron posibles debido a la expropiación de los territorios, las culturas y las sabidurías de los pueblos originarios (Quijano, 2000). La colonialidad del poder de los estados en América Latina persiste, ya que no ha surgido un «proceso de decolonización de las relaciones sociales, políticas y culturales que han mantenido y reproducido la clasificación social» (Quijano, 2000, p. 568). El énfasis se pone en la «colonialidad», referida «a los patrones de poder de larga duración que han surgido como resultado del colonialismo, pero que definen la cultura, el trabajo, las relaciones intersubjetivas y la producción de conocimientos» que persiste hasta el día de hoy (Maldonado-Torres, 2007, p.243). De manera similar, los investigadores indígenas provenientes de los estados colonizados argumentan que las políticas de Estado reproducen las estructuras del poder colonial y continúan subordinando a los pueblos indígenas (Coulthard, 2014; Tuhiwai Smith, 2012). 
Es importante evaluar las suposiciones implícitas que subyacen a las normas y políticas, tales como la Ley 27811. Es especialmente importante para mí, como alguien no indígena y no peruana, reflexionar acerca de mi postura con respecto a la reproducción de la colonialidad del poder y de los conocimientos.

Durante doce meses, entre 2012 y 2013, trabajé con el equipo de Sociodiversidad del Instituto de Investigaciones de la Amazonía Peruana-San Martín (IIAP-SM), asistiendo a las comunidades indígenas en San Martín, Perú, para que registrasen sus conocimientos ecológicos tradicionales (CET) a través de la Ley 27811. Trabajé como voluntaria por medio de Uniterra, un programa canadiense de desarrollo internacional que opera junto con el World University Service of Canada (WUSC) y el Centre d'Étude et de Coopération Internationale (CECI) (organizaciones canadienses independientes de cooperación internacional) y quienes reciben financiación del gobierno de Canadá a través de Global Affairs Canada ${ }^{1}$.

Mi rol como voluntaria, al ser empleada y financiada por Uniterra, fue ayudar a Sociodiversidad a lograr sus objetivos y a liderar actividades complementarias. El IIAP-SM asistió a nueve comunidades nativas ${ }^{2}$ (cuatro awajún ${ }^{3}$ y cinco kichwa lamistas ${ }^{4}$ ) a registrar y proteger sus conocimientos colectivos a través de la Ley 27811. Sociodiversidad fue el nexo entre cada comunidad y el Instituto Nacional de Defensa de la Competencia y de la Protección de la Propiedad Intelectual (Indecopi), organismo responsable de administrar la ley ${ }^{5}$. Con el objetivo de documentar la sabiduría indígena, se elaboraron asambleas comunitarias y grupos de discusión. En las comunidades kichwa que habían iniciado el proceso de registro, ayudé con las actividades para darles seguimiento y fui responsable de guiar a tres comunidades awajún en el proceso de su inscripción. Viví en San Martín hasta mediados de 2014 y posteriormente continué visitando el lugar de manera regular (ocho viajes en ocho meses). A pesar de no estar relacionado con mi trabajo para el IIAP, mi constante contacto con los líderes indígenas regionales y con una comunidad kichwa me proporcionaron el contexto y la oportunidad de observar los impactos duraderos de la ley en la comunidad.

1 El análisis y opiniones expresados en este artículo son de la autora y no representan las opiniones de las organizaciones mencionadas.

2 El término «comunidad nativa» es un término legal que refiere a las comunidades indígenas amazónicas en el Perú.

Awajún es el nombre preferido actual; anteriormente y alternativamente aguaruna.

Alternativamente kechwa, y antiguamente, quechua lamista.

Indecopi es una institución gubernamental independiente responsable por la promoción de un mercado justo y honesto. 
San Martín se ubica en la selva alta en el norte de Perú. Tiene una larga historia con el Estado colonial: su capital, Moyobamba, fue fundada por los españoles en 1540. La Amazonía peruana también tiene una larga historia de extracción de recursos: barbasco (veneno de pez), caucho, maderas, petróleo, coca (Aramburú, 1982; Calderón, 2003). Los pueblos indígenas amazónicos han sido marginados y continúan perdiendo territorio a causa de los migrantes, las áreas de conservación y los intereses extractivistas (Calderón, 2003; Greene, 2009). Los indígenas kichwa lamistas estuvieron sujetos a trabajos forzados al haber perdido varias campañas militares a mediados de los 1600 (Regan, 1993). Permanecen en relaciones económicas desiguales con los mestizos (Calderón, 2003; Calle, 2014) ${ }^{6}$.

Los kichwa lamistas sostienen relaciones de cuidado con plantas, animales y espíritus que habitan sus territorios, tanto en sus jardines como en el bosque (Panduro y Rengifo, 2001; Regan, 1993). Poseen un extenso conocimiento acerca de las plantas medicinales (Brown, 2014). A pesar de que no fueron inmunes al colonialismo español, los awajún evitaron la conquista española y recientemente - desde la mitad del siglo XX - han empezado a asentarse en poblados de manera más sedentaria con la influencia de la iglesia evangélica y la educación bilingüe (Brown, 2014; Calle, 2014; Greene, 2009). Numerosos awajún en San Martín ahora viven cerca de la Carretera Marginal, la vía principal que conecta la Amazonía nororiental con la costa. Los awajún también conservan sus jardines de horticultura y poseen conocimientos acerca de muchas plantas medicinales; varios espíritus de las plantas, comestibles y medicinales, deben ser aplacados a través de ícaros (anen en awajún) (Brown, 2014; Guallart, 1989).

Conocidos como guerreros, los awajún han movilizado su aprendizaje del bilingüismo hacia la abogacía por sus derechos de manera directa, para enfrentar al Estado. Al día de hoy, los pueblos indígenas en San Martín están bien organizados políticamente. Las federaciones representan a los grupos de comunidades nativas (tanto las que ya son reconocidas legalmente como las que están por serlo) que pertenecen al mismo grupo etnolingüístico en determinada área de la región. Dichas federaciones son socias de la federación panétnica regional, que a su vez está afiliada a la Asociación Interétnica de Desarrollo de la Selva Peruana (Aidesep), con sede en Lima.

6 Mestizos son los descendientes de los conquistadores españoles y los pueblos originarios. Véase De la Cadena (2000) para una descripción de mestizaje y la relación entre mestizos y pueblos indígenas. 
La Ley 27811 tiene como objetivo proteger el «conocimiento colectivo» de los pueblos originarios, definido en el artículo $2 \mathrm{~b}$ como un «conocimiento acumulado y transgeneracional desarrollado por los pueblos y comunidades indígenas respecto de las propiedades, usos y características de la diversidad biológica». Los CET se refieren a este tipo de sabiduría ecológica que se ha compartido de manera intergeneracional (Taubman y Leistner, 2008). El término CET es debatible, porque infiere que el conocimiento es estático y promueve la visión eurocéntrica de que los humanos pueden estar separados de su medio ambiente (Nadasdy, 1999). Yo uso el término como abreviatura, ya que la Ley 27811 únicamente protege los conocimientos colectivos relacionados con el medio ambiente, pero no otros tipos de legado cultural.

Los CET como concepto obtuvieron popularidad en la década de 1990, porque podían ser incorporados a marcos de referencia científicos cartesianos (Nadasdy, 1999). Los marcos de referencia científicos cartesianos se refieren a las ciencias «modernas» y sus conceptos claves son los dualismos y la racionalidad (Plumwood, 1993). Descartes contribuyó al desarrollo de los dualismos al separar la mente del cuerpo. Los dualismos se basan en las jerarquías, con una categoría dominante y el resto subordinadas (Plumwood, 1993). Quijano (2000) argumenta que los europeos, al basarse en los dualismos, se veían a sí mismos como la sociedad más avanzada (y racional) del mundo. Todas las otras historias fueron homogeneizadas como primitivas, cercanas a la naturaleza, y por lo tanto, irracionales. Los pueblos indígenas alrededor del mundo poseen tecnologías sofisticadas que los poderes coloniales europeos usaron para avanzar en sus propios métodos (en la minería, por ejemplo) (Quijano, 2000). El uso del término «ciencia cartesiana» reconoce que no es el único método científico posible: se pueden producir conocimientos fidedignos sin tener que privilegiar a la humanidad por encima del resto de la naturaleza.

Yo argumento que la Ley 27811 es un mecanismo legal poderoso, con el potencial de tener un efecto más allá del reconocimiento simbólico, pero que no altera los diferenciales del poder, los cuales permanecen ahí cuando se incorpore un sistema de conocimientos a otro. Para hacer explícitos los supuestos que hay detrás de la Ley 27811, la he contextualizado dentro de los acuerdos internacionales. Continúo con una crítica a la presentación de dicha ley y a las limitaciones de su implementación: al enfocarse en las amenazas externas, no aborda las desigualdades internas dentro del Estado-nación. Proporciona protecciones a los DPI de los pueblos indígenas peruanos, pero no existe evidencia de que se logren sus objetivos de desarrollo. Los pueblos indígenas peruanos dan 
prioridad a los derechos territoriales y a su derecho a tener un consentimiento previo e informado acerca de los proyectos de desarrollo. Respetar los DPI de los pueblos indígenas es importante, pero es tan solo un aspecto de una lucha más amplia por su reconocimiento y sus derechos sobre la tierra.

\section{LAS PROTECCIONES INTERNACIONALES DE LOS CET}

Las discusiones para proteger sistemáticamente a los CET resultaron en partes del Convenio sobre Diversidad Biológica (CDB) y la creación, por la Organización Mundial de la Propiedad Intelectual (OMPI) del Comité Intergubernamental sobre Propiedad Intelectual y Recursos Genéticos, Conocimientos Tradicionales y Folclore (CIG). El artículo 8j del CDB - al estipular que los gobiernos deben proteger, preservar y mantener los conocimientos, las innovaciones y las prácticas de los pueblos indígenas en relación con el uso y conservación de la diversidad biológica - es un reconocimiento internacional fundamental de los CET (Brown, 2003; Clark, et al., 2004; Hayden, 2003; Taubman y Leistner, 2008). Además de protección, el CDB requiere que los Estados-nación proporcionen mecanismos de acceso al patrimonio genético dentro de sus fronteras (Hayden, 2003).

Los discursos simultáneos del acceso y de la protección «entablan conexiones débiles entre el capitalismo y la conservación» (Muehlmann, 2007, p. 18). El CDB convirtió la biodiversidad en un objeto que puede ser identificado, documentado, protegido y explotado (Hayden, 2003; Muehlmann, 2007). A pesar de que el artículo 8j reconoce a los CET, pone énfasis en el potencial para conservar la biodiversidad, en lugar de valorar a los pueblos indígenas por sí mismos (Muehlmann, 2007). Al convertir la biodiversidad en un objeto, el CDB y la Ley 27811 refuerzan «sistemas de recursos intramuros» y la posibilidad de propiedad privada sobre la naturaleza que se ha visto en los DPI convencionales (Drahos, 2014, p. 85).

Esta unión de acceso y protección fue característica de muchas negociaciones de bioprospección durante las décadas de 1990 y 2000. Posey (1996), un partidario por el control de los pueblos indígenas sobre sus conocimientos, fomentó la bioprospección como oportunidad para el desarrollo sostenible. La bioprospección se refiere al «desarrollo corporativo de medicinas basadas en plantas, el conocimiento tradicional y la obtención de microorganismos de las regiones "ricas en biodiversidad" alrededor del mundo» (Hayden, 2003, p. 1). Las ganancias están aseguradas por patentes que pertenecen a las compañías farmacéuticas, pero que están basadas en el conocimiento local acerca de sus recursos. Patentar formas de 
vida biológicas ha sido posible gracias a la decisión de la corte estadounidense de Diamond vs. Chakrabarty: los genes modificados genéticamente, alterados por ingeniería genética o que han sido aislados al ser obtenidos de un recurso biológico se han vuelto patentables (Brown, 2003; Hayden, 2003). La bioprospección tiene el potencial de obtener impactos positivos, pero los críticos argumentan que es un proyecto colonial, porque se continúa obteniendo ganancias de las personas marginadas (LaDuke, 2005; Mgbeoji, 2006; Tedlock, 2006; Tuhiwai Smith, 2012; Whitt, 2009). Poniendo énfasis en la apropiación de los saberes, algunos se han referido a la bioprospección como biopiratería: el «patentar los saberes indígenas relacionados con la biodiversidad» (Shiva, 2007, p. 307). Patentar las formas de vida biológicas no solo deniega la creatividad humana, sino también la creatividad de la naturaleza (Shiva, 2001).

Los acuerdos de distribución de beneficios financiados por el Grupo de Cooperación Internacional de la Biodiversidad (ICBG, por sus siglas en inglés) son quizá los acuerdos de bioprospección más destacados, los que conectan las compañías farmacéuticas con los interesados. Aunque sean bienintencionados, se ha comprobado que son controversiales; la RAFI (Rural Advancement Foundation International) - una ONG canadiense - ha argumentado que ICGB estaba cometiendo biopiratería (Brown, 2003; Greene, 2002, 2004; Hayden, 2003). Los esfuerzos de la RAFI en contra de la biopiratería contribuyeron a la cancelación de un proyecto de ICBG en México y estuvieron a punto de cancelar ICGB-Perú (Brown, 2003; Hayden, 2003). El proyecto cancelado fue un esfuerzo de colaboración, producto de una relación de larga duración con algunos mayas en México. Los antropólogos que lideraban el proyecto después argumentaron que «la oposición al proyecto fue, en efecto, una oposición a la autonomía local de la comunidad sobre el control para acceder a sus recursos biológicos» (Berlin y Berlin, 2004, p. 473).

El contrato original de ICGB-Perú fue con el Consejo Aguaruna-Huambisa, una federación indígena que representa a varias comunidades awajún. Después de que RAFI proporcionó una copia del convenio de licencia entre la Universidad de Washington y Searle — que no incluía al consejo—, retiraron su apoyo al proyecto. Los pueblos indígenas, sin embargo, no son un grupo homogéneo: una federación rival, la Organización Central de Comunidades Aguarunas del Alto Marañón (OCCAAM), inició negociaciones formales con ICBG en 1995 (Greene, 2002, 2004). La OCCAAM eligió a César Sarasara —entonces presidente del grupo nacional por los derechos de los pueblos amazónicos indígenas llamado Confederación de Nacionalidades Amazónicas del Perú (CONAP) como el mediador del proyecto. Sarasara, y un equipo de abogados negociaron 
un convenio de licencia de know-how con el gigante farmacéutico Searle, donde se permitía a los awajún conservar la propiedad sobre sus CET. César Sarasara acredita esta asociación exitosa como precursora de la Ley 27811, porque «se convirtió en el modelo a partir del cual un marco de referencia regulatorio [para la Ley 27811] fue diseñado» (Clark et al., 2004, p. 773; Sarsara, 2010). A pesar de que es un acuerdo legal ejemplar, no ha impulsado a la compañía Searle a desarrollar medicinas nuevas y ha dejado a los awajún sin ninguna de las regalías esperadas. Searle no renovó el acuerdo, que expiró en el año 2000 (Greene, 2004).

Dos acuerdos regionales hechos por la Comunidad Andina - una organización intergubernamental — también propiciaron la creación de la Ley 278117. En 1996, la Comisión de la Comunidad Andina aprobó la Decisión 391 que establece el «Régimen Común sobre Acceso a los Recursos Genéticos». Esta ley interestados requiere que las patentes basadas en CET cuenten con acuerdos por escrito por los respectivos poseedores de conocimientos (Ortega, 2014; Taubman y Leistner, 2008). En el año 2000, la Comunidad Andina estableció un «Régimen Común de Propiedad Intelectual», la Decisión 489. El artículo 3 demanda que los Estadosmiembros se aseguren de que los DPI respeten el patrimonio biológico y genético y los conocimientos tradicionales de las comunidades indígenas, afroamericanas y locales. Con respecto a la Decisión 391, esta reitera que las patentes derivadas de CET no pueden ser otorgadas sin el permiso explícito de las comunidades de origen. Estas leyes internacionales están directamente relacionadas con la Ley 27811 del Perú.

\section{LALEY 27811:RÉGIMEN DE PROTECCIÓN DE LOS CONOCIMIENTOS COLECTIVOS DE LOS PUEBLOS INDÍGENAS VINCULADOS CON LOS RECURSOS BIOLÓGICOS}

Esta ley sui generis es, en principio, un sistema de registro, y está considerada como la «mejor elaborada» de su tipo (Taubman y Leistner, 2008). Cuenta con tres registros: el local, el público y el confidencial. Es responsabilidad de una comunidad el iniciar y mantener un registro local para su propia referencia. Los registros locales no son enfatizados en los materiales informativos de la ley, y nosotros tampoco los enfatizamos cuando ayudamos a las comunidades en su proceso de registro. El Indecopi mantiene los registros públicos y confidenciales, usados para anular patentes o patentes en trámite alrededor del mundo. Muchos

7 Los miembros de la Comunidad Andina son Bolivia, Colombia, Ecuador y Perú. 
de los conocimientos tradicionales son del «dominio público», abiertamente conocidos o divulgados en publicaciones previas ${ }^{8}$. Este tipo de información se inscribe en el registro público para ser usada como evidencia de «arte anterior». Esto significa que una patente basada en conocimientos registrados no puede ser nueva y no puede ser válida. Para defender el mal uso de los CET, que aún no son muy conocidos, el registro de confidencialidad de la Ley 27811 no está disponible de manera pública y tampoco se puede buscar. No puede usarse como evidencia de arte anterior, porque al hacerlo se divulgaría la información de manera pública; sí puede, en cambio, emplearse como evidencia para usurpar legitimidad a alguien que se proclame inventor de la patente sin realmente serlo. Esto facilita que haya una apelación a la patente sin necesidad de exponer a la fuente original de conocimientos (Ortega, 2014; Taubman y Leistner, 2008).

El Indecopi no otorga patentes de CET a las comunidades; los conocimientos registrados reciben títulos. Los títulos no aplican para un individuo, sino para un colectivo comunal. Solicitar una patente es costoso, pero desde el año 2006, registrar los CET es gratuito para las comunidades (El Peruano, 2006). Múltiples comunidades pueden registrar el mismo uso de igual recurso, y el registro no los priva de compartir el conocimiento entre comunidades. Una vez que las comunidades hayan acumulado sus conocimientos, el Indecopi registra a cada uno de manera individual y lo clasifica en los registros públicos o confidenciales.

Investigadores y compañías que estén en busca de usar CET proveniente de los pueblos indígenas del Perú requieren consentimiento previo e informado por una federación indígena que represente a varias comunidades. La Ley 27811 establece un Fondo para el Desarrollo de los Pueblos Indígenas, a quien las compañías deben contribuir con el 10\% de las ganancias de sus ventas en caso que se estén capitalizando al usar CET del Perú. Las compañías también deben contribuir a la financiación si obtienen ganancias de CET del dominio público en caso que este se haya hecho público después del año 1982. Esta financiación tiene como objetivo generar ingresos y asegurar la repartición de beneficios equitativos de los productos que se hayan desarrollado a partir de los CET.

Indecopi estableció en 2004 la Comisión Nacional contra la Biopiratería, responsable de anular patentes existentes y patentes en trámite alrededor del mundo9. La comisión utiliza los registros y una lista priorizada de 35 especies

Véase Hayden (2003) para una discusión de la creación del «dominio público».

Anteriormente, la Comisión Nacional para la protección al acceso a la diversidad biológica peruana y a los conocimientos colectivos de los pueblos indígenas. 
nativas del Perú (en su mayoría de flora) para buscar peticiones en las oficinas internacionales de patentes. Posteriormente, interpone demandas legales en contra de la patente. Entre otras, han prevenido exitosamente que una patente francesa utilizara el aceite de la nuez sacha inchi en sus cosméticos. La solicitud de la patente reconoció que los pueblos indígenas peruanos utilizan sacha inchi para la piel clara. Fue voluntariamente retirada por la compañía solicitante porque la Comisión desafió las afirmaciones de ser esta una patente nueva y habló directamente con la compañía afrontada (Biopiratería, 2017). Su trabajo demuestra acciones concretas tomadas en contra de la biopiratería, pero que no otorgan un beneficio concreto para los pueblos indígenas. Su énfasis está proteger el patrimonio peruano en lugar de aquel que pertenece a alguna comunidad indígena.

\section{EL DERECHO CONSUETUDINARIO}

La Ley 27811 se presenta a sí misma como si respetara el derecho consuetudinario de los indígenas. Para dar inicio al proceso de registro, una comunidad debe autorizar su permiso a través de una asamblea general. Dicha junta comunitaria incluye un voto por familia, generalmente representado por el varón, jefe del hogar. Esto opaca los desbalances de poder (especialmente la desigualdad de género) dentro de las comunidades. A pesar de su rol en generar y mantener la biodiversidad, las voces de las mujeres a menudo están ausentes (Brown, 2014; Mgbeoji, 2006). En el seguimiento correcto del derecho consuetudinario de los pueblos indígenas se respeta su soberanía (Brown, 2003). Se vuelve problemático cuando determinado modelo del derecho consuetudinario se aplica a través de políticas y prioridades coloniales de producción sobre una diversidad de tradiciones. En el Perú, lo que se considera derecho consuetudinario forma parte de una estructura de toma de decisiones impuesta a través de leyes de titulación de tierras indígenas.

En 1974, el gobierno peruano aprobó la Ley 22175, Ley de Comunidades Nativas y de Desarrollo Agrario de la Selva y Ceja de Selva ${ }^{10}$. Otorga tenencia de la tierra a comunidades indígenas de la Amazonía, mientras que asegura la soberanía nacional y promueve el desarrollo económico a través de la agricultura. Para recibir un título, una comunidad debe adoptar una estructura de liderazgo específica, incluyendo un presidente, un vicepresidente y un tesorero. Las deci-

10 Esta ley, ratificada en 1978, reemplazó la ley original de 1974 (Ley 20653, Ley de Comunidades Nativas y de Promoción Agropecuaria de la Selva y Ceja de Selva). 
siones se toman en las comunidades en una asamblea general. La Ley de las Comunidades Nativas tomó como modelo un proceso de titulación de tierras andinas que comenzó por 1920 (Greene, 2009). Las comunidades indígenas (principalmente las andinas) se pudieron registrar como tales pidiendo al gobierno «certificados de reconocimiento oficial a territorios de propiedad colectiva de origen colonial (los indios solicitantes tenían que demostrar que habían ocupado sus tierras "desde tiempos inmemorables"), en donde los miembros cultivaban la tierra de manera individual y criaban a sus animales en los pastizales naturales de la comunidad» (Mayer, 2009, p. 10) ${ }^{11}$. Cuando las comunidades nativas recibieron un título legal por los años 1970, recibían visitas de los trabajadores del gobierno para asegurarse de que eligieran representantes para ocupar las posiciones de liderazgo indicadas: Brown (2014) hace el recuento de cómo los burócratas visitaron dos veces en solo un mes a una pequeña comunidad awajún para que hubiera elecciones.

Ya que el derecho consuetudinario en el que la Ley 27811 se basa para seguir su proceso de registro también fue legislado y aplicado por el Estado peruano, podría considerarse como una imposición del Estado, aunque esto último es discutible. Richland $(2008,2011)$ destaca la complejidad para identificar lo que se considera gobernabilidad tradicional, argumentando que las evaluaciones de la tradición deben de provenir de los mismos pueblos indígenas. Las contradicciones son inherentes en el derecho consuetudinario legislado para todos los grupos indígenas, pero aún más en aquel que haya sido adoptado activamente. Greene (2009) argumenta que los awajún adecuan el concepto de indigenidad para hacer valer su soberanía y competir por el poder de manera estratégica. Las comunidades pueden ser habilidosas para adaptar estructuras legales de gobernabilidad y acomodarlas a cambios de liderazgo repentinos, por ejemplo.

Dos comunidades awajún a las que asistimos a través del proceso de registro pasaron por revueltas de liderazgo en 2012 y 2013. En una de ellas, las autoridades gubernamentales del Perú se vieron en la necesidad de involucrarse; sin embargo, la comunidad pudo participar en el proceso de registro porque el consejo usurpado se formalizó antes del día oficial de registro ${ }^{12}$. En otra de las comunidades, Indecopi se presentó para registrar formalmente los conocimientos colectivos, pero en lugar de eso, fue testigo de la expulsión del presidente de la

\footnotetext{
11 A partir de 1969, las comunidades indígenas andinas fueron reconocidas como comunidades campesinas, en un esfuerzo para minimizar el perjuicio contra «indios».

12 Véase Brown (2014) para una breve explicación del inicio del conflicto mencionado.
} 
comunidad. El segundo caso fue menos complicado —el vicepresidente asumió la presidencia - pero hubo una demora en el proceso, que no pudo continuar sino hasta que el nuevo liderazgo se hubiera formalizado. Mientras que los pueblos indígenas pueden adaptar temporalmente el derecho consuetudinario impuesto para su beneficio, estos ejemplos también muestran que se pueden ver restringidos por su estructura.

\section{DIFICULTADES EN LA PRESENTACIÓN DE LA LEY}

En sus materiales de divulgación, la ley perpetúa estereotipos erróneos acerca de los pueblos indígenas y la bioprospección. En la antigua página web de la ley, la parte de la introducción romantiza a los pueblos indígenas al presentar imágenes de personas con vestimentas tradicionales dando el mensaje de que «su contacto con la naturaleza los convierte en poseedores de una sabiduría invaluable, que es nuestro deber protegerlo y promover su uso justo y equitativo» (Indecopi, 2017). Aunque es importante notar que dicha página introductoria ya no está vigente, los pueblos indígenas fueron presentados como si estuvieran congelados en el tiempo, no como actores políticos competentes y organizados. El texto hace una evocación al «noble salvaje», que se refiere a la idea de que los pueblos indígenas viven en armonía con la naturaleza. Algunos critican esta idea como demasiado simplista, equiparando a los pueblos indígenas como si fueran parte de la naturaleza y mostrándolos como menos que humanos (Brosius, 1997). Esto representa de manera equivocada lo que muchos contextualizan como relaciones de parentesco y responsabilidades con lo no humano (De la Cadena, 2015; Salmón, 2000; TallBear, 2011; Todd, 2017). Muchos pueblos indígenas sí cuentan con conocimientos extensos basados en su lugar de origen y pueden ejercer un rol en la conservación, pero esta contextualización opaca los diversos métodos científicos que continúan generando CET. Con esto se recalca la dicotomía implícita de que los no indígenas pueden estar separados del medio ambiente (mientras que los indígenas no pueden). Bajo este esquema no existe un espacio para reconocer a los indígenas que cuentan con educación universitaria, y esto perpetúa las ideas coloniales de que los pueblos indígenas son irracionales (Quijano, 2000). Tanto en el Perú como en otros lados, los indígenas han sido considerados como niños, ignorantes e incapaces de ser conscientes de las consecuencias de sus actos. Aún hoy, muchos peruanos no indígenas consideran a los pueblos indígenas como retrógradas y no educados (Calderón, 2003; De la Cadena, 2000). 
Los materiales de divulgación de la ley están basados en ideas populares acerca de la biopiratería; por ejemplo, la compañía farmacéutica occidental que explota a los pobladores marginados de los países en vías de desarrollo. Esto se encuentra en los folletos de Indecopi que explican por qué la ley es necesaria y cómo funciona. Uno de ellos es una tira cómica que ilustra a un hombre blanco y rubio con pecas, vestido con traje de safari y sombrero Tilley, formulando preguntas a una persona local acerca del uso de las plantas. Este personaje engaña a la comunidad, obteniendo ganancias a partir de los conocimientos adquiridos. Aunque esto contribuye a las ideas populares acerca de la bioprospección, la realidad es más compleja. Hayden (2003) describe cómo los científicos sondean los costados de las carreteras y los mercados buscando medicinas, en lugar de sondear a los poblados. Además, las compañías farmacéuticas hoy en día tienen muy poco interés en buscar CET (Brown, 2003). Los CET son mucho más valiosos para la industria de la agricultura, y también más propensos a ser sujetos de biopiratería. A menudo, grandes compañías patentan variedades de cultivos - tales como quinoa y frejoles provenientes de los Andes-, que son producidas por pequeños agricultores (McAfee, 2008). La quínoa, desarrollada y cosechada por pueblos indígenas andinos, es patentada por la nación-estado peruana junto con la Universidad de Colorado (Tedlock, 2006). A pesar de que muchos indígenas peruanos practican la horticultura y sus conocimientos sobre sus recursos han sido patentados por otros en el Perú y en otras partes del mundo, los materiales de divulgación de la ley únicamente pusieron énfasis en los conocimientos sobre recursos médicos.

Existe un desbalance de poderes entre los investigadores extranjeros y las comunidades indígenas, pero estas representaciones enturbian las imposiciones políticas dentro de las fronteras del Perú. Greene (2002) y Coombe (1998) argumentan que los pueblos indígenas por lo general son marginados con mayor frecuencia por los estados-nación que por fuerzas externas. En 2009, a un costado de la Carretera Marginal, grupos indígenas liderados por los awajún y los huambisas hicieron un bloqueo carretero a modo de protesta en contra de una legislación gubernamental que permitía la apertura de la cuenca amazónica a la extracción. Las comunidades con las que trabajamos estuvieron presentes en el «baguazo» - un joven líder kichwa me dijo recientemente: «estábamos todos ahí», a pesar de que para muchos el lugar les quedaba a varias horas hacia el este sobre la carretera. El bloqueo se encontraba en una curva peligrosa de la carretera llamada La Curva del Diablo, cerca del poblado de Bagua. La legislación gubernamental transgredía sus derechos legales a un consentimiento previo e informado. En ese caso, el entonces presidente, Alan García los llamó «perros 
del hortelano», insinuando que los pueblos indígenas estaban estorbando el desarrollo por no utilizar los recursos de la tierra ni permitir que otros accedieran a ellos. El 5 de junio, por razones que permanecen inciertas, se desató la violencia entre la policía y los indígenas que estaban manifestándose. El «baguazo» logró su objetivo al cambiar la legislación ${ }^{13}$; sin embargo, es un poderoso recordatorio de lo que está en riesgo y un ejemplo de cómo el Estado peruano puede poner en peligro el derecho de los indígenas a un consentimiento previo e informado.

Al introducir la ley en las comunidades indígenas, a menudo hacíamos representaciones de la tira cómica descrita con anterioridad, para explicar de manera simple y efectiva cómo funciona la ley y qué es lo que hace. Necesitábamos utilizar los materiales aclaratorios que tuviéramos al alcance, suministrados por Indecopi. Poner énfasis en los conocimientos medicinales en los materiales de divulgación contribuye a un mayor enfoque sobre este tipo de conocimiento en el registro. Si nosotras, como promotoras de la ley, hubiéramos tenido mayor conocimiento de los matices del problema, habríamos estado mejor preparadas para apoyar a las comunidades a través del proceso.

\section{DIFICULTADES EN EL PROCESO DE IMPLEMENTACIÓN}

A pesar de que fue ratificado en 2002, el proceso de registro de la ley comenzó en 2006, cuando se volvió gratuito para las comunidades. La implementación comenzó lentamente: Indecopi otorgó 92 títulos de un total de 193 solicitudes comunitarias en cuatro años, y 99 títulos fueron «abandonados» (Indecopi, 2009). Desde entonces, Indecopi formó alianzas estratégicas con organizaciones peruanas, incluyendo el IIAP. Como enlace y socio estratégico, el rol del IIAP era el de acumular información para ser registrada y coleccionar especímenes biológicos. Indecopi solo puede visitar a las comunidades participantes en el único día del registro oficial. Los especímenes deben ser recolectados para que los biólogos del IIAP puedan identificar sus nombres científicos, esenciales para el proceso de registro. En diciembre de 2013, Indecopi organizó una ceremonia en San Martín para presentar de manera simbólica los 629 títulos registrados para ocho comunidades (faltaba procesar a la novena comunidad) (Calle, 2014). Para el año 2017, Indecopi había otorgado 4893 títulos, la mayoría en el registro confidencial (Andina, 2018) ${ }^{14}$.

13 Véase Brandenburg y Orzel (2016) por un resumen e introducción a la protesta.

14 Hasta mediados de 2020, Indecopi había otorgado 6585 títulos (PCM, 2020). 
Solo una fracción de las comunidades nativas y campesinas del Perú han participado en el proceso hasta ese momento: menos de la mitad del porcentaje de comunidades indígenas en Perú están representadas en el registro del conocimiento colectivo de Indecopi. De acuerdo con un reporte del 2016, existen 8164 comunidades indígenas diferentes. De ellas, 1667 no cuentan con un título o reconocimiento legal. Esto incluye a 1023 comunidades campesinas y 644 comunidades nativas (Smith y Salazar, 2016). Indecopi ha buscado activamente promover la ley y expandir sus protecciones, pero no es factible que todas las comunidades indígenas (con o sin título) puedan participar en el proceso.

El trabajo comunitario del IIAP es importante y contribuye a que más CET se puedan registrar bajo la Ley 27811. Sin embargo, estos títulos únicamente representan una parte del conocimiento que poseen los miembros de las comunidades. En preparación para el Indecopi, el IIAP organizó un día de talleres comunitarios. En un poblado kichwa, las personas llenaron la casa comunal. Algunos traían consigo plantas, que fueron fotografiadas para facilitar su identificación taxonómica. Dividimos a las personas en grupos, cada uno con hojas de rotafolio y marcadores. Se les dio aproximadamente dos horas para que hicieran una lista de todas las especies que se les ocurrieran, incluyendo sus usos y preparaciones. Esta dinámica sucedió de manera similar en cada comunidad; una mujer awajún estaba automedicándose con cushingilla ${ }^{15}$, aplicando la ortiga sobre sus piernas hábilmente mientras el resto del grupo consideraba hacer otras preparaciones. A pesar del trabajo del IIAP con las comunidades para identificar otros usos de los recursos biológicos (identificando tintes naturales, por ejemplo), la información recolectada en los talleres comunitarios explícitamente para el proceso de registro se enfocó en el tratamiento de lesiones y enfermedades. Cuando un grupo se atascaba en el proceso, una etnobotánica les preguntaba, por ejemplo, cómo tratar un malestar estomacal. Por lo general este incentivo era suficiente para reactivar un grupo.

El enfoque en las plantas medicinales no solo es un producto de la pericia disponible para asistir con los talleres: también refleja la abrumadora preocupación acerca de la bioprospección farmacéutica. Los incentivos generan discusiones, pero no representan todas las condiciones que pueden ser tratadas con recursos locales: existe un límite para recordar, a petición, conocimientos que a menudo

15 Algunos nombres y detalles identificadores de personas, comunidades y recursos biológicos (si no forman parte del conocimiento común y previamente publicado) han sido cambiados para proteger la anonimidad de participantes. 
están implícitos. Además, no podíamos garantizar la participación de todos los miembros de la comunidad. El Alto Mayaku parecía entusiasmado por comenzar el proceso (leer a continuación), pero el día del taller el salón se sentía vacío: solo tuvimos participantes para formar tres grupos pequeños. No siempre es posible para los individuos renunciar a un día de trabajo en el campo para asistir a un taller comunitario. En otra de las comunidades, un hombre joven y su anciano padre me preguntaron por qué no podíamos visitar a su padre en su hogar. El señor conocía muchos anen y era un chamán habilidoso. Desafortunadamente, el IIAP estaba limitado en cuanto a recursos (de presupuesto, personal y tiempo). Así, la información recolectada en los talleres, y posteriormente registrada a través de la Ley 27811, no representa la profundidad de conocimientos en determinado lugar.

\section{LA TRADUCIBILIDAD Y LA DENOMINACIÓN}

En sus procesos de registro, la Ley 27811 subordina a los CET a la ciencia cartesiana, como muestran los problemas relacionados con la traductibilidad: el lenguaje, la taxonomía y la extractabilidad. Con respecto a los problemas de traducción, Nadasdy (2003) y Cruikshank (2005) cuestionan cuán eficientemente se pueden incorporar los CET a estrategias de cogestión. Dado que los CET forman parte de un sistema de conocimiento holístico, un aspecto no puede $-\mathrm{y}$ en teoría no debe- ser aislado y colocado fuera de contexto. Argumentan que cuando se incorporan los CET y la ciencia cartesiana a un marco de referencia de cogestión, un sistema de conocimientos inevitablemente subordina al otro. Los CET son valorados y reconocidos de manera superficial, pero únicamente aquellos elementos que puedan ser extraídos y aceptados con facilidad por la ciencia cartesiana son integrados a una estrategia.

Tratar la información como algo alienable y extraíble es contrario a percibir a los CET como parte de un sistema holístico y reafirma marcos de referencia de ciencia cartesiana. Como ya se ha mencionado, la Ley 27811 separa a los CET de otras expresiones culturales; pobladores de las comunidades con quienes trabajamos lamentaban esta limitación. En lugar de recibir un reconocimiento por su riqueza en conocimientos, la ley está diseñada para aislar los recursos individuales: a cada preparación le es otorgado un título. La ley y sus estadísticas intentan contabilizar lo que no es contable (Muehlmann, 2012). Únicamente si se les considera en su totalidad, los conocimientos registrados pueden comenzar a aproximarse a las ricas tradiciones intelectuales del Perú. 
La problemática de la traductibilidad es tal vez más evidente cuando se requieren nombres científicos. Previamente he mencionado que 99 solicitudes de titulación fueron abandonadas. Esto no se refiere a un título rechazado sino a un nombre científico que hace falta en el registro. Una comunidad cuenta con seis meses para proveer un nombre científico faltante antes de que el proceso se considere como abandonado. En estos casos, una comunidad es responsable económicamente de identificar muestras biológicas, con lo cual es posible que se vean limitados a continuar progresando. Como socio estratégico y vínculo del Indecopi, el IIAP ha identificado y otorgado nombres científicos para los requisitos de los títulos. Meses después del registro oficial, el Indecopi informó a mi colega que faltaban algunos de los nombres científicos. Ella dedicó un fin de semana a recolectar especímenes en una comunidad, y después tuvo que conseguir los fondos para pagarle a un experto y que los identificara.

En un país en donde se hablan múltiples lenguas indígenas, y con el objetivo de anular patentes internacionales, se necesita un sistema de nombramiento unificador. Sin embargo, los nombres científicos no pueden y no capturan otros sistemas categóricos, y que se requiera nombres científicos cuando tal vez no los haya significa que el registro falla en una función importante: el documentar y proteger los conocimientos de los recursos que la ciencia aún no reconoce. Winona LaDuke (2005, p. 76) argumenta que «la cultura dominante ha mantenido una persistente tendencia hacia el querer descubrir, clasificar y recolectar todo lo que se considere exótico en términos convencionales». Se considera que en la Amazonía se encuentra el 15\% de la biodiversidad mundial y que contiene varias especies aún no descubiertas y sin nombre científico, a pesar de que ya las reconocen los pobladores locales (Brown, 2003). Si una de estas especies se toma como muestra para hacer un registro, un científico tendrá la oportunidad de nombrarla. En un comunicado al OMPI, una organización de indígenas peruanos llamado Centro de Culturas Indígenas del Perú (Chirapaq) pone énfasis en la problemática que hay, pues los científicos se apropian de sus conocimientos y recursos, se los llevan a los laboratorios y les asignan nombres nuevos (RiveraZea, 2006). La clasificación para asignar nombres que sigue los lineamientos del sistema Linnaean es una forma de imperialismo lingüístico; los nombres de las plantas kichwas incluyen información cultural. Schiebinger (2004, p. 197) argumenta que «precisamente este tipo de información — la del uso medicinal, la distribución biogeográfica, y la validez cultural - era usurpada de las plantas en la nomenclatura binominal que maneja el sistema Linnaean». Ngũgĩ wa Thiong'o (1986) explica cómo el lenguaje es colonizador, y este hecho resulta 
en una lucha por tener relevancia. ¿Los nombres de quién son reconocidos? ¿A quién se le otorga el derecho de nombrar?

Privilegiar a los nombres científicos da prioridad a un sistema taxonómico. Mientras nos enseñaba su jardín, un líder awajún nos explicaba cómo algunos awajún pueden reconocer y distinguir veintitrés tipos diferentes de jengibre. Tenía sembradas dieciocho variedades, pero esperaba poder sembrarlas todas. Cada una tiene un nombre awajún diferente y sirve para tratar distintas partes del cuerpo. Cada uso tiene un título propio, pero todas están registradas bajo el mismo nombre científico. Este ejemplo también demuestra la adaptabilidad de los CET, ya que el jengibre es una especie introducida. Los mismos métodos para aprender acerca de la flora nativa — en este caso, la habilidad para percibir las diferencias energéticas - fueron aplicados para poder diferenciar a las plantas de jengibre. La Ley 27811 únicamente protege al producto de este método científico, aislándolo como un fragmento de información. Este énfasis privilegia al conocimiento sobre los portadores de ese conocimiento (Muehlmann, 2007). El requisito de tener nombres científicos indica que los CET deben ser legitimados a través de marcos teóricos cartesianos, reafirmando la colonialidad del poder.

\section{LA RECEPCIÓN DE LA LEY EN LAS COMUNIDADES INDÍGENAS}

Al salir de la Carretera Marginal y tomar una calle de tierra con palmeras enfiladas, pasamos a través del retén del poblado hacia el Alto Mayaku. Mi colega y yo estamos ahí para explicar la Ley 27811. Ya habíamos hablado acerca de ella con los líderes de la comunidad el mes anterior; ahora estamos asistiendo a una reunión de la asamblea general para introducir la ley y sus objetivos a toda la comunidad nativa. Un gran edificio de cemento sin adornos ya estaba lleno de gente sentada en bancas de madera. Mi colega y yo nos paramos al frente. Los líderes de la comunidad se sentaron en la mesa hacia nuestra izquierda, de frente a los demás miembros de la comunidad. Después de nuestra presentación, los invitamos a hacernos preguntas. Un hombre que estaba cerca de la parte de enfrente se puso de pie y le habló a la audiencia en awajún. Hablaba con pasión y gesticulaba enfáticamente. Yo me sentí incómoda, interpreté su tono como enojado, y creo que lo escuché mencionar la palabra «gringos». Al ser una extranjera de piel blanca, tomé conciencia de que mi presencia podría ser interpretada de manera negativa. Ni mi colega — una peruana mestiza - ni yo hablamos awajún. Para nuestro beneficio, uno de los líderes tradujo los puntos principales del discurso pronunciado por el hombre: estos extranjeros vienen aquí, se llevan 
nuestro conocimiento como si fuera su investigación y no regresan. Después de un par de comentarios más, nos pidieron que preparáramos un acta formal para empezar el proceso de los registros de su comunidad para que los representantes de hogares pudieran firmar inmediatamente. Estábamos sorprendidas: esperábamos que un acta que diera consentimiento al proceso de registro de manera oficial y colectiva sería firmada en una reunión posterior, como había ocurrido en las otras comunidades con las que estábamos trabajando. Nos fuimos de ahí sorprendidas, pero animadas de poder comenzar el proceso rápidamente en el Alto Mayaku.

Los líderes indígenas y las comunidades consideran esta ley como un reconocimiento simbólico de su identidad y sus derechos. Ellos buscan activamente herramientas políticas para asegurar que sus derechos sean respetados, y esta ley protege su derecho a un consentimiento previo e informado, específicamente de cómo sus conocimientos de los recursos biológicos pueden ser usados por otros. Como se ha mencionado con anterioridad, la Comisión Nacional Anti-Biopiratería ha tenido algunas victorias en obstruir patentes basadas en la flora peruana, a pesar de que al parecer no existan ganancias monetarias para el fondo nacional establecido, y supuestamente ha habido problemas para iniciar el comité de gestión que se haría cargo de supervisar el fondo y de decidir cómo usarlo. Aún es muy pronto para juzgar su efectividad de poder acumular ganancias para las comunidades. Greene (2002) argumenta que el «verdadero peligro» del ICBG-Perú son las expectativas incumplidas de concesiones financieras, para las cuales esta ley no ofrece protección. Muchas comunidades indígenas se encuentran dentro de los grupos más empobrecidos económicamente del país y por tanto tienen la necesidad de ingresos monetarios; una promesa de paga a cambio de CET puede parecer muy atractiva. Sin embargo, como se ha mencionado, la paga a menudo no se materializa. Por lo tanto, «se corre el riesgo de que se haga más profundo el sentido de empobrecimiento y traición que sienten los aguarunas en manos de los investigadores y los intereses privados» (Greene 2002, p. 242); esto puede aplicar para otros pueblos indígenas.

La ley puede ayudar a protegerlos del mal uso de los conocimientos, pero no puede disuadir a los investigadores de obtener conocimientos de estos lugares y desaparecer, como lo lamentaba el hombre mencionado con anterioridad. Él criticaba a los investigadores por no regresar, algo que es común que suceda después de que se termina un proyecto de desarrollo o de investigación. Esto va más allá de los DPI y saca a relucir críticas más amplias con respecto al trabajo de desarrollo y otros proyectos a corto plazo con grupos marginados. 
A pesar de que la ley fue bien recibida en las comunidades con las que trabajamos - uno de los líderes nos agradeció cuando terminamos el trabajo porque él creía que era importante-, no tiene un impacto en la vida diaria de las personas. El otro trabajo del IIAP se enfocaba en la formación de capacidades. En visitas subsecuentes, la gente podría mencionar estos otros proyectos, pero nadie menciona sus títulos registrados. Es un instrumento legal abstracto que no contribuye a su supervivencia diaria. Existe cierto peligro de que el proceso de registro y sus beneficios se atrofien después de que los títulos hayan sido otorgados. La ley es un instrumento legal importante internacionalmente, pero sus efectos no son percibidos de manera local.

\section{CONCLUSIÓN}

Como instrumento legal, la Ley 27811 puede abogar en contra del mal uso de los CET en el ámbito internacional, aunque esconda desigualdades nacionales. Reconoce de manera simbólica a los pueblos indígenas del Perú, pero va más allá del simbolismo por el rol activo que tiene el Estado de promover la ley y por el financiamiento para convertirla en un proceso gratuito. Los problemas remarcados con anterioridad demuestran el propósito de la ley, que está escrita para una audiencia legal occidental en lugar de estar escrita para los pueblos indígenas directamente. La Ley 27811 es reguladora de las vidas indígenas; coloca a los CET dentro de un marco teórico universal, para convertirlos en «otra esfera de actividad regulada» (Brown, 2003, p. 214). Continúa dando prioridad a la ciencia cartesiana por encima de los métodos indígenas. Fracasa como mecanismo de desarrollo y no aborda la necesidad de apoyar económicamente a las comunidades empobrecidas. Al defenderlos únicamente contra los intereses internacionales, la Ley 27811 no reconoce los agravios hechos en contra de los pueblos indígenas desde adentro de las fronteras nacionales. La explotación hecha por cualquier élite, incluyendo la élite del Estado, necesita impedirse.

A pesar de que la Ley 27811 refuerza los derechos que tienen los pueblos indígenas a ejercer un consentimiento previo e informado, no cambia la subyacente relación de subordinación entre los pueblos originarios y el Estado. Este tipo de leyes otorgan protecciones importantes dentro del sistema existente, y por esta razón, vale la pena apoyarlas. Sin embargo, es importante que el trabajo no se detenga ahí porque no facilite a los pueblos indígenas mejores herramientas para administrar todos sus recursos. Los indígenas del Perú consideran que sus mayores riesgos son las amenazas a su territorio. En San Martín existe la tala 
ilegal, la posibilidad de que se implemente la exploración petrolera y los migrantes que se asientan de manera continua en territorio indígena. Algunas comunidades tienen muy pocos recursos, porque siguen esperando que el Estado les otorgue su título oficial. Más allá de las protecciones a los CET, el Estado tiene que tomar acciones concretas para reconocer la soberanía de los pueblos indígenas dentro de sus fronteras ${ }^{16}$.

\section{REFERENCIAS BIBLIOGRÁFICAS}

Andina (25 de enero de 2018). Indecopi entrega 700 registros de conocimientos colectivos a comunidades indígenas. Andina: Agencia Peruana de Noticias. Recuperado de https://andina.pe/agencia/noticia-indecopi-entrega-700-registros-conocimientos-colectivos-a-comunidades-indigenas-697217.aspx

Asher, Kiran. (2013). Latin American Decolonial Thought, or Making the Subaltern Speak. Geography Compass, 7(12), 832-842. https://doi.org/10.1111/gec3.12102

Aramburú, Carlos Eduardo. (1982). Expansión de la frontera agraria y demográfica de la selva alta peruana. En C. Aramburú, E. Bedoya Garland y J. Recharte Bullard (eds.), Colonización en la Amazonía (pp. 1-39). Lima: Centro de Investigación y Promoción Amazónica.

Berlin, Brent y Berlin, Elois Ann. (2004). Community Autonomy and the Maya ICBG Project in Chiapas, Mexico: How a Bioprospecting Project that Should Have Succeeded Failed. Human Organization, 63(4), 472-486. https://doi.org/10.17730/ humo.63.4.xp2dpuh204b0h61b

Brandenburg, Heidi y Orzel, Mathew. (2016). When Two Worlds Collide. Documental. Lima: Yachaywasi Films.

Brosius, J. Peter. (1997). Endangered Forest, Endangered People: Environmentalist Representations of Indigenous Knowledge. Human Ecology, 25(1), 47-69. https://doi.org/110.1023/A:1021983819369

16 Nota: después de la publicación en inglés de este artículo, en una visita a una de las comunidades nativas con conocimientos registrados bajo la Ley 27811, la autora ha notado que, en la casa comunal del pueblo, habían colocado el título simbólico. Esta acta, tomada siete años después de que terminó el proceso de registro, demuestra que, en algunos casos, los líderes indígenas recuerdan y hacen recordar de todas las herramientas que reconocen sus derechos. Colocar el título simbólico demuestra que los pueblos indígenas en el Perú —aunque algunos parecen remotos - conocen sus derechos bajo las leyes nacionales e internacionales. A la vez, no cambia las conclusiones del artículo: los títulos otorgados a cada comunidad nativa no cambian sus vidas diarias. 
Brown, Michael F. (2003). Who Owns Native Culture? Cambridge, MA: Harvard University Press.

Brown, Michael F. (2014). Upriver: The Turbulent Life and Times of an Amazonian People. Cambridge, MA: Harvard University Press.

Calderón Pacheco, Luis Alberto. (2003). Relaciones interétnicas entre mestizos y nativos kechwa en Lamas en el contexto de la globalización. En C.I. Degregori (ed.), Comunidades locales y transnacionales: cinco estudios de caso en el Perú, (pp. 13-104). Lima: IEP.

Calle Roalcaba, Orly Denisse. (2014). Conocimientos tradicionales vinculados a los recursos biológicos y su protección en las comunidades nativas de la región San Martín. Iquitos: IIAP.

Clark, Susanna, Lapeña, Isabel y Ruiz, Manuel. (2004). The Protection of Traditional Knowledge in Peru: A Comparative Perspective. Washington University Global Studies Law Review, 3(3), 755-797.

Comisión Nacional contra la Biopiratería (Biopiratería) (2017). Comisión Nacional contra la Biopiratería. Recuperado de http://www.biopirateria.gob.pe/ (8 de abril 2017).

Coombe, Rosemary. (1998). The Cultural Life of Intellectual Properties: Authorship, Appropriation, and the Law. Durham, NC: Duke University Press.

Coulthard, Glen. (2014). Red Skin, White Masks: Rejecting the Colonial Politics of Recognition. Minneapolis: University of Minnesota Press.

Cruikshank, Julie. (2005). Do Glaciers Listen? Local Knowledge, Colonial Encounters, and Social Imagination. Vancouver: University of British Columbia Press.

De la Cadena, Marisol. (2000). Indigenous Mestizos: The Politics of Race and Culture in Cuzco, Peru, 1919-1991. Durham, NC: Duke University Press.

De la Cadena, Marisol. (2015). Earth Beings: Ecologies of Practice across Andean Worlds. Durham, NC: Duke University Press.

Drahos, Peter. (2014). Intellectual Property, Indigenous People and their Knowledge. Cambridge, United Kingdom: Cambridge University Press.

El Peruano (2006). Modifican texto único de procedimientos administrativos del Instituto Nacional de Defensa de la Competencia y de la Protección de la Propiedad Intelectual. Resolución administrativa 076-2006-PCM.

Greene, S. (2002). Intellectual Property, Resources, or Territory? Reframing the Debate over Indigenous Rights, Traditional Knowledge, and Pharmaceutical Bioprospection. En M.P. Bradley y P. Petro (eds.), Truth Claims: Representation and Human Rights (pp. 229-249). New York: Rutgers. 
Greene, Shane. (2004). Indigenous People Incorporated? Culture as Politics, Culture as Property in Pharmaceutical Bioprospecting. Cultural Anthropology, 45(2), 211237. https://doi.org/10.1086/381047

Greene, Shane. (2009). Customizing Indigeneity: Paths to a Visionary Politics in Peru. Stanford, CA: Stanford University Press.

Guallart, Jose Maria, S.J. (1989). El mundo mágico de los aguarunas. Lima: CAAAP.

Hayden, Cori. (2003). When Nature Goes Public: The Making and Unmaking of Bioprospecting in Mexico. Princeton, NJ: Princeton University Press.

Indecopi (2009). El registro de conocimientos colectivos de los pueblos indigenas asociados con la biodiversidad. Informe. Lima: Indecopi.

Indecopi (2017). Ley 27811. Recuperado de http://servicio.indecopi.gob.pe/portalctpi/ (17 de febrero 2017).

LaDuke, Winona. (2005). Recovering the Sacred: The Power of Naming and Claiming. Cambridge, MA: South End Press.

Maldonado-Torres, Nelson. (2007). On the Coloniality of Being: Contributions to the Development of a Concept. Cultural Studies, 21(2-3), 240-270. https://doi. org/10.1080/09502380601162548

Mayer, Enrique. (2009). Ugly Stories of the Peruvian Agrarian Reform. Durham, NC: Duke University Press.

McAfee, Kathy. (2008). Exporting Crop Biotechnology: The Myth of Molecular Miracles. En G. Otero (ed.), Food for the Few: Neoliberal Globalism and Biotechnology in Latin America (pp. 61-90). Austin: University of Texas Press.

Mgbeoji, Ikechi. (2006). Global Biopiracy: Patents, Plants and Indigenous Knowledge. Vancouver: University of British Columbia Press.

Mignolo, Walter D. (2011). The Darker Side of Western Modernity: Global Futures, Decolonial Options. Durham, NC: Duke University Press.

Muehlebach, Andrea. (2001). «Making Place» at the United Nations: Indigenous Cultural Politics at the UN, Working Group on Indigenous Populations. Cultural Anthropology, 16(3), 415-448. https://doi.org/10.1525/can.2001.16.3.415

Muehlmann, Shaylih. (2007). Defending Diversity: Staking Out a Global, Common Interest? En A. Duchene y M. Heller (eds.), Discourses of Endangerment: Ideology and Interest in the Defence of Languages (pp. 14-34). New York: Bloomsbury Publishing.

Muehlmann, Shaylih. (2012). Rhizomes and Other Uncountables: The Malaise of Enumeration in Mexico's Colorado River Delta. American Ethnologist, 39(2), 339-353. https://doi.org/10.1111/j.1548-1425.2012.01368.x 
Nadasdy, Paul. (1999). The Politics of TEK: Power and the «Integration» of Knowledge. Arctic Anthropology, 36(1/2), 1-18. https://www.jstor.org/stable/40316502

Nadasdy, Paul. (2003). Hunters and Bureaucrats: Power, Knowledge, and Aboriginal-State Relations in the Southwest Yukon. Vancouver: University of British Columbia Press.

Ortega, Aurora. (2014). Marco regulatorio y base legal relacionado al acceso a los recursos genéticos y protección de conocimientos tradicionales. I Seminario Regional de Protección e Innovación en Productos Naturales de Origen Peruano. Lima: Indecopi.

Panduro, Rider. y Rengifo, Grimaldo. (2001). Montes y montaraces: la visión del bosque en los quechua-lamas. Una aproximación. Lima: Proyecto Andino de Tecnologías Campesinas.

Plumwood, Val. (1993). Feminism \& the Mastery of Nature. New York: Routledge.

Posey, Darrell. (1996). Protecting Indigenous Peoples' Rights to Biodiversity. Environment, 38(8), 6-9, 37-45.

Presidencia del Consejo de Ministros (2020). Orgullo peruano: hay 6585 conocimientos colectivos de pueblos indígenas registrados. Nota de prensa. 30 dejulio. Recuperado de https://www.gob.pe/institucion/indecopi/noticias/217883-orgullo-peruanohay-6585-conocimientos-colectivos-de-pueblos-indigenas-registrados.

Quijano, Anibal. (2000). Coloniality of Power, Eurocentrism, and Latin America. Nepantla: Views from South, 1(3), 533-580.

Regan, Jaime, S.J. (1993). Hacia la tierra sin mal: la religión del pueblo en la Amazonía. Iquitos: Centro de Estudios Teológicos de la Amazonía.

Richland, Justin. (2008). Arguing with Tradition: The Language of Law in Hopi Tribal Court. Chicago, IL: The University of Chicago Press.

Richland, Justin. (2011). Hopi Tradition as Jurisdiction: On the Potentializing Limits of Hopi Sovereignty. Law \& Social Inquiry, 36(1), 201-234. https://www.jstor.org/ stable/23011875

Rivera-Zea, Tarcila. (2006). El conocimiento tradicional en la visión indígena. Comunidades locales e indígenas: preocupaciones y experiencias en la promoción, el manteamiento y la protección de sus conocimientos tradicionales y sus recursos genéticos. Panel de la 10ª sesión del Comité Intergubernamental de la Organización Mundial de la Propiedad Intelectual, Geneva, Switzerland, 30 de noviembre.

Salmón, Enrique. (2000). Kincentric Ecology: Indigenous Perceptions of the Humannature Relationship. Ecological Applications, 10(5), 1327-1332. https://doi. org/10.1890/1051-0761(2000)010[1327:KEIPOT]2.0.CO;2 
Sarasara, César. (2010). Consentimiento fundamentado previo y libre: experiencias comunitarias en las esferas de los recursos genéticos, los conocimientos tradicionales y las expresiones culturales tradicionales: Las experiencias de Perú. Panel de la 16 $6^{\text {a }}$ sesión del Comité Intergubernamental de la Organización Mundial de la Propiedad Intelectual, Geneva, Switzerland, 3-7 de mayo.

Schiebinger, Londa. (2004). Plants and Empire: Colonial Bioprospecting in the Atlantic World. Cambridge, MA: Harvard University Press.

Shiva, Vandana. (2001). Protect or Plunder? Understanding Intellectual Property Rights. Halifax, Canada: Fernwood Publishing Ltd.

Shiva, Vandana. (2007). Bioprospecting as Sophisticated Biopiracy. Signs: Journal of Women in Culture and Society, 32(2), 307-313. https://doi.org/10.1086/508502

Smith, Richard Chase, y Salazar, Milagros. (2016). Tierras comunales: más que preservar el pasado es asegurar el futuro: el estado de las comunidades indígenas en el Perú. Report. Lima: Instituto del Bien Común.

TallBear, Kim. (24 de abril 2011). Why Interspecies Thinking Needs Indigenous Standpoints. Theorizing the Contemporary. Cultural Anthropology. Recuperado de https:/culanth.org/fieldsights/260-why-interspecies-thinking-needs-indigenousstandpoints

Taubman, Antony, y Leistner, Matthias. (2008). Traditional Knowledge. En von S. Lewinski (ed.), Indigenous Heritage and Intellectual Property: Genetic Resources, Traditional Knowledge, and Folklore. Segunda edición (pp. 59-179). Alphen aan den Rijn, the Netherlands: Kluwer Law International.

Tedlock, Barbara. (2006). Indigenous Heritage and Biopiracy in the Age of Intellectual Property Rights. Explore: The Journal of Science and Healing, 2(3), 256-259.

Thiong'o, Ngũgĩ wa. (1986). Decolonizing the Mind: The Politics of Language in African Literature. Portsmouth, NJ: Heinemann.

Todd, Zoe. (2017). Fish, Kin and Hope: Tending to Water Violations in amiskwaciwâskahikan and Treaty Six Territory. Afterall: A Journal of Art, Context and Enquiry, 43(1), 102-107. https://doi.org/10.1086/692559

Tuhiwai Smith, Linda. (2012). Decolonizing Methodologies: Research and Indigenous Peoples. 2nd ed. New York: Zed Books.

Varese, Stefano. (1996). The New Environmentalist Movement of Latin American Indigenous People. En S. Brush y D. Stabinksy (eds.), Valuing Local Knowledge: Indigenous People and Intellectual Property Rights (pp. 112-142). Washington, DC: Island Press.

Whitt, Laurelyn. (2009). Science, Colonialism, and Indigenous Peoples: The Cultural Politics of Law and Knowledge. Cambridge, UK: Cambridge University Press. 